Neurosurg Focus 11 (3):Article 5, 2001, Click here to return to Table of Contents

\title{
Motor cortex stimulation
}

\author{
JEFFrey A. Brown, M.D. \\ Department of Neurological Surgery, Wayne State University, School of Medicine, Detroit, Michigan
}

\begin{abstract}
In 1991 Tsubokawa and colleagues first published their landmark results from a series in which epidural motor cortex stimulation (MCS) was used in the treatment of eight patients with central and neuropathic pain. In ensuing studies authors have elaborated on the indications, technique, hypotheical mechanisms, and beneficial results of this treatment. Epidural MCS is effective for trigeminal neuropathy, lateral medullary and thalamic infarction, anesthesia dolorosa, postherpetic neuralgia, spinal cord injury, and limb stump pain. Postoperative outcomes are better when patients present with only mild or absent motor weakness in the region of pain and when there is pain in the trigeminal region. It is hypothesized that MCS is effective because it increases regional cerebral blood flow in the ipsilateral ventrolateral thalamus in which corticothalamic connections from the motor and premotor areas predominate. The extent of pain alleviation also correlates with the increase of blood flow in the cingulate gyrus. This suggests that stimulation reduces the suffering experienced by a patient with chronic pain. Procedure-related morbidity has included epidural hematoma, subdural effusion, gradual diminution of benefit, and painful stimulation. Although of concern, treatment-induced chronic seizure disorders have not occurred as a complication or in animal models of chronic cortical stimulation. Stimulation-induced pain relief occurs within minutes. There are no associated paresthesias or muscle contractions that confirm function. Pain relief may last for hours after electrical stimulation is discontinued. Motor cortex stimulation is an established therapy for the treatment of complex central and neuropathic pain syndromes that have proved refractory to medical treatment.
\end{abstract}

KEY WORDS • motor cortex stimulation • pain

\section{HISTORY OF ELECTRICAL STIMULATION FOR PAIN SYNDROMES}

Central pain is defined by the International Association for the Study of Pain as pain initiated or caused by a primary lesion or dysfunction in the central nervous system.

The earliest observations that led to work in this area was made by Penfield and Jasper. ${ }^{13}$ During their studies of patients with epilepsy, they observed that stimulation of the precentral gyrus elicited sensory responses when the corresponding portion of the adjacent postcentral gyrus was resected. Additionally, in 1954, they reported the treatment results of a patient with unilateral burning pain that was relieved after removal of the contralateral postcentral gyrus. When the pain recurred, it was again relieved, this time after resection of the precentral gyrus. ${ }^{13}$ As Lende and associates ${ }^{13}$ have noted these observations led to the understanding that both the post- and precentral gyri are involved in pain. In 1955, White and Sweet ${ }^{27}$ wrote of their attempted surgical treatment to cure central pain. They performed postcentral gyrectomy. Only 13\%

Abbreviations used in this paper: $\mathrm{CBF}=$ cerebral blood flow; $\mathrm{ES}=$ electrical stimulation; $\mathrm{MCS}=$ motor cortex stimulation; $\mathrm{SCI}=$ spinal cord injury; VAS $=$ visual analog scale. of their patients continued to experience pain relief. Lende and associates ${ }^{13}$ published case reports of patients with central neuropathic facial pain in whom long-term pain relief was achieved by resection of the precentral and adjacent postcentral facial cortex.

At about this time, in another approach, Hosobuchi ${ }^{9,10}$ hypothesized that stimulation of the neospinothalamicleminiscal system, the system that mediates nonpainful stimuli, could inhibit pain. He treated patients with facial anesthesia dolorosa by undertaking thalamic stimulation as well as stimulation of the internal capsule. In 1983, Andy ${ }^{1}$ used electrical stimulation of the thalamus to treat central pain syndromes. Using thalamic recordings, he first identified spontaneous discharge sites that were located predominantly in the centromedian-parafascicular complex and related intralaminar nuclear structures, and then blocked the activity by using ES. Shortly thereafter, in $1985, \mathrm{Namba}^{18}$ also found that facilitation of the medial lemniscus inhibited pain, perhaps by inhibiting spinothalamic activity. He noted that lemniscal and extralemniscal (spinothalamic) systems intermingle in the thalamic and suprathalamic levels. Namba, et al., ${ }^{19}$ also found deafferentation hyperactivity in the subnucleus caudalis of the spinal trigeminal nucleus in cats after performing gasserian ganglionectomy. Along the same lines, Tsubokawa, 


\section{J. A. Brown}

et al. ${ }^{25,26}$ performed retrogasserian rhizotomy in cats and stimulation of the thalamic sensory relay nucleus, and studied the effects of this treatment on deafferentation pain. They found that trigeminal medullary dorsal horn hyperactivity was inhibited. Because of all this work, thalamic involvement in central pain became an established concept.

In 1985, however, Hardy and Haigler ${ }^{8}$ returned to study the cortical level. They stimulated the medial prefrontal cortex in rats and found a significant elevation of the nociceptive response latency by using hot-plate and tail-flick techniques. No seizures occurred. Stimulation of control sites did not cause any similar increase in latency. They concluded that stimulation of the prefrontal cortex can provide pain relief. ${ }^{7,8}$ Hosobuchi ${ }^{10}$ implanted electrodes in the subcortical somatosensory region in 44 patients for the treatment of dysesthetic chronic pain. Leg pain was more effectively treated by stimulation of the somatosensory region than by stimulation of the periaqueductal gray area. In 1989 Lenz, et al., ${ }^{14}$ found that the somatosensory thalamic neurons in patients with SCI-induced central pain fire more frequently than do similar neurons in patients without such pain. Using microelectrode recording and microstimulation, Gorecki, et al., ${ }^{6}$ found that, in the deafferented thalamus, a phenomenon of central allodynia was identified in which patients with hyperpathia experience pain on stimulation rather than the usual sensations. These studies were undertaken to identify the most appropriate site for treatment of central pain by using electrophysiological techniques.

In 1991, Tsubokawa, et al., ${ }^{23,24,26}$ introduced epidural MCS as a less invasive alternative treatment for central deafferentation pain. They endeavored to determine a safer procedure than deep brain stimulation, which they found to be associated with significant morbidity. These authors discovered that ES of the motor cortex also inhibited thalamic burst activity. They treated seven patients with thalamic pain by performing MCS with epidural plate electrodes. Good to excellent pain control was obtained in all cases, and the authors reported no complications or side effects. During the stimulation, regional CBF increased to both the cortex and thalamus. Temperature in the painful skin regions rose, and there was improved movement observed in the painful limbs. When the postcentral gyrus was stimulated, pain was either exacerbated or unchanged. Patients underwent evaluation for more than 2 years after surgery. The effect of precentral gyrus stimulation was unabated in five patients; the effect diminished over the course of several months in the other three patients. No seizures were observed, and there was no electroencephalographic evidence of any seizure activity. Pain relief was achieved using stimulus intensities below the threshold for muscular movement. ${ }^{23}$ The authors had now established that MCS was a viable therapy for central pain.

In subsequent studies the authors have elaborated on the indications, technique, hypothetical mechanisms, and beneficial results of this form of treatment. Epidural MCS has been found to be effective in the treatment of trigeminal neuropathy, lateral medullary infarction, anesthesia dolorosa, and postherpetic and glossopharyngeal neuralgia. In more recent and extensive series long-term follow- up results have been documented., 3,12,17,22 In one series of 10 patients, when the central fissure was localized by combining stereotactic magnetic resonance imaging with somatosensory evoked potentials, a $40 \%$ pain relief rate was achieved in $50 \%$ of the cases. ${ }^{20}$ Katayama, et al., ${ }^{11}$ reviewed the treatment of 31 patients for whom 2-year follow-up data were available. Good or excellent pain relief ( $>60 \%$ pain reduction) was achieved in $48 \%$ of the patients. In $73 \%$ of patients in whom there was no evidence of motor weakness good to excellent results were achieved. When motor contractions could not be induced, only $9 \%$ of the patients $(\mathrm{p}<0.01)$ experienced pain relief. It appears that an intact corticospinal tract neuronal system originating from the motor cortex is necessary for effective pain relief. ${ }^{11}$

Since 1991 the use of MCS has been focused on the treatment of neuropathic facial pain. Nguyen, et al. ${ }^{20}$ have reported on 10 patients with neuropathic facial pain followed for more than 2 years in whom substantial pain relief $(75 \%$ and $77 \%)$ was achieved. Ebel, et al., ${ }^{3}$ reported on a series of patients with trigeminal neuropathic pain syndromes (anesthesia dolorosa and postherpetic neuralgia). Using a VAS three of six patients described pain reduction of more than 50\% that was maintained for 6 months to 2 years. In the other three patients the benefit gradually decreased over several months. ${ }^{3}$ The number of MCS-treated patients discussed in the literature has since been increasing exponentially. Since 1995, the procedure has become popularized worldwide and is now a part of standard literature and teaching practices used in the field of neurosurgery.

\section{MOTOR CORTEX STIMULATION}

\section{Mechanism of Effect}

Positron emission tomography studies demonstrate that cortical stimulation increases $\mathrm{CBF}$ in the ipsilateral thalamus, cingulate gyrus, orbitofrontal cortex, and brainstem. In one patient in whom long-lasting pain relief was reported, a greater increase in CBF was observed in the thalamus and brainstem; an intact somatosensory system does not appear to be necessary to receive benefit from cortical stimulation. ${ }^{21}$ Further, the authors observed that regional $\mathrm{CBF}$ increases occur in the ipsilateral ventrolateral thalamus in which corticothalamic connections from the motor and premotor areas predominate. Regional CBF increases in this site, however, are less than those seen in the anterior cingulate gyrus, insula, and brainstem. There is a correlation with the extent of pain relief and the increase in cingulate blood flow. This finding suggests that stimulation improves the suffering component of patients with chronic pain manifested in the Papez circuit. Activation of the brainstem periaqueductal gray area is also a possible effect. $^{5}$ The results of these CBF studies confirm that the somatosensory cortex is not activated by MCS, nor are there changes in the flow through the motor pathways below the stimulating electrode.

One group has observed that MCS attenuates brainstem spinal pain reflexes. ${ }^{7}$ Although an intact somatosensory system is not required for successful treatment, the presence of an intact corticospinal tract neuronal system orig- 
inating from the motor cortex is required for pain relief to be effective. As previously stated, Katayama, et al., ${ }^{11}$ have noted that pain relief was satisfactory in $73 \%$ of patients with mild or absent motor weakness. When motor weakness was present and was moderate to severe, there was therapeutic benefit in only $15 \%$ of the 13 patients. When motor contractions could not be induced, pain relief was achieved in only $9 \%$ of patients $(\mathrm{p}<0.01){ }^{11}$

\section{Clinical Indications}

Motor cortex stimulation should be considered for the treatment of central neuropathic pain syndromes that have failed to respond adequately to medical therapy. Neuropathic pain is characterized by allodynia, dysesthesias, paresthesias, and hypalgesia and should be distinguished from nociceptive pain. Patients who are likely to benefit from the procedure may experience at least $40 \%$ pain relief with intravenous thiamylal when given in increments of $50 \mathrm{mg}$ every 5 minutes to a maximum dose of $250 \mathrm{mg}$. They are less likely to respond to intravenous morphine when given in $0.3-\mathrm{mg}$ increments every $5 \mathrm{~min}$ utes to a maximum of $18 \mathrm{mg} .{ }^{28}$ Syndromes that have been treated by MCS include anesthesia dolorosa and other forms of trigeminal deafferentation pain, central pain secondary to stroke, postherpetic neuralgia, peripheral deafferentation pain syndromes such as brachial plexus or sciatic nerve injury, SCI, and phantom limb and stump pain. Patients who also suffer dense weakness in the painful area are less likely to benefit from this procedure.

\section{Preoperative Evaluation}

To characterize the neuropathic nature and severity of the patient's pain, it is helpful to administer a preoperative McGill Pain Questionnaire and a VAS. ${ }^{15,16}$ In neuropathic pain, the dysesthesias are often characterized as constant and burning. The McGill Pain Questionnaire and VAS can be used postoperatively to monitor the patient's progress.

Because the decision to proceed with permanent implantation of the MCS device is based on a lengthy screening process, patients must be capable of adequately communicating with health care workers as to the effects and effectiveness of alterations in their stimulation parameters. To date, the United States Food and Drug Administration has approved the electrodes implanted in this procedure for human use and labeled marketing for spinal pain applications. Currently, cortical implantation represents an "off-label" application in the United States.

\section{Operative Technique}

In their early description of the procedure Tsubokawa, et al., ${ }^{23}$ and Meyerson, et al., ${ }^{17}$ performed the operation after administration of a local anesthetic. Since then the technique has evolved. General anesthesia may be used instead because of the accuracy of target planning in which intraoperative three-dimensional image-guided neuronavigation is used.

Preoperatively, fiducials are fixed to the scalp and a magnetic resonance image is obtained using 1-mm cuts. Computerized tomography scanning may also be used. The contralateral central sulcus, Sylvian fissure, and inferior and superior frontal sulci are identified. Optimal re- sults for facial pain are obtained when the target is identified anterior to the central sulcus adjacent to or below the inferior frontal sulcus. In cases of upper-limb pain, the best results are obtained when the selected target is anterior to the central sulcus in the mid precentral region.

Electromyography electrodes are inserted for intraoperative monitoring after ES, as are electrodes for median nerve stimulation. Prophylactic antibiotic medication is given along with anticonvulsant agents. After induction, muscle relaxants are stopped to allow for electromyography monitoring of muscular stimulation. The patient is placed in a lateral or supine position with a cloth roll placed beneath the shoulder and the head rotated to the ipsilateral side of the pain. The target is mapped onto the contralateral scalp and a linear incision delineated that lies over the central sulcus. The draping procedure allows for a wide enough scalp exposure so that the electrode wire can exit behind the ear and for observation of facial movement during intraoperative cortical stimulation, if facial pain is to be treated. The incision is long enough to allow for a circular craniotomy opening with an approximate minimum diameter of $5 \mathrm{~cm}$.

Once the craniotomy flap is turned, any dural openings are sutured closed, and a 16 to 20 -plate diagnostic electrode array is placed in the epidural space aligned parallel to the line of the central sulcus, covering both the regions of the pre- and postcentral gyri.

Median nerve somatosensory evoked potential recordings are obtained to identify the N20/P20 phase shift that occurs across the central sulcus. This provides electrophysiological confirmation of the site of the precentral gyrus in the region of the hand, located cephalad to the facial region. The physiological data confirm the anatomical targeting performed using neuronavigation. With experience, it may not be necessary. The target for electrode implantation is marked on the dural surface. The facial motor region is then stimulated using the grid electrodes. Stimulation is performed in a train of three at $200-\mu \mathrm{sec}$ durations and less than $20 \mathrm{~mA}$. The side of the face contralateral to the pain is observed for contractions in cases of facial pain. The electromyography electrodes are placed in appropriate muscle groups to monitor for muscular contractions.

When the appropriate cortical target is confirmed by motor stimulation, the diagnostic grid is removed, and a flat, four- or eight-plate electrode is positioned perpendicular to the central sulcus (Fig. 1). The central two electrodes cross the identified target. In cases of thalamic infarction syndrome, two electrode paddles may be implanted where the region of pain is more extensive, covering a wider region of the motor cortex. Each electrode is sutured to the outer, periosteal layer of the dura, and the lead wire is tunneled out through a separate stab incision. The craniotomy bone flap is secured.

The electrode can be tested as soon as the patient is fully alert. Testing should continue for several days or until the patient can consistently confirm that stimulation reduces the preoperative pain by at least $50 \%$.

A second craniotomy, also after induction of general anesthesia, is required for permanent implantation of a pulse generator, which is usually positioned in a pocket in the upper chest wall and connected to the electrode. A gener 


\section{J. A. Brown}

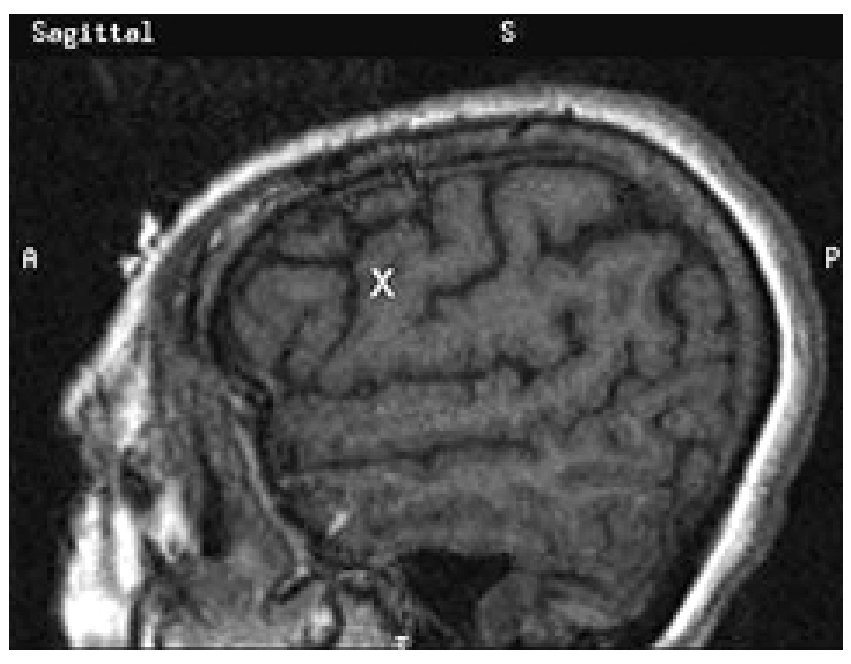

Fig. 1. Sagittal magnetic resonance demonstrating the target site (X) for treatment in a patient with neuropathic facial pain. The target is located anterior to the central sulcus and posterior to the splitting of the inferior frontal sulcus.

ator capable of connecting to one or two four-electrode arrays may be implanted.

\section{Stimulation Parameters}

Stimulation is generally performed at a frequency range of 25 to $55 \mathrm{~Hz}$, pulse width of 60 to $180 \mu \mathrm{sec}$, and amplitude of 1.3 to $4 \mathrm{~V}$. Higher voltage (up to $7 \mathrm{~V}$ ) has been advocated for use in patients with anesthesia dolorosa. Bipolar stimulation is used with the negative pole overlying the motor cortex and positive pole over the sensory cortex. Pain relief may last beyond the period of stimulation. Nguyen, et al., ${ }^{20}$ have used a pattern consisting of 3 hours of stimulation alternating with 3 hours off. Others have used shorter intervals of "on" stimulation. The benefit of stimulation appears to occur within minutes. There may also be a period in which pain relief endures after simulation is discontinued.

Often intraoperative MCS-induced pain relief is evident in the recovery room. Usually, patients are only able to determine that the stimulator is turned on because they feel relief from their pain. In some cases paresthesias can be projected onto the same area of distributions as the pain. In other cases headache in the region in which the cortical electrode was placed has occurred. This improves after the stimulus intensity is decreased without reducing the clinical benefit.

\section{Procedure-Related Morbidity}

Motor cortex stimulation-related complications reported include intraoperative seizures, stimulator-pocket infection, epidural hematoma, subdural effusion, gradual diminution of pain relief benefit over a period of several months, and dehiscence of the stimulator pocket. In one patient in an early series, an epidural hematoma developed after the dura was denervated in a second operation performed to reduce painful sensations occurring at the dural electrode implantation site. ${ }^{17}$

Chronic seizures after implantation of an MCS elec- trode have not been observed. In 1999 Bezard, et al., ${ }^{2}$ studied the effect of cortical stimulation in three healthy monkeys. Using frequency and pulse duration of $40 \mathrm{~Hz}$ and $90 \mathrm{msec}$, respectively, and an intensity set just below the threshold for eliciting muscle twitch, seizures were not induced. Increasing the stimulus intensity did induce reversible epileptic seizures; however, the threshold for these did not change even after seizures were induced.

\section{SUMMARY}

Motor cortex stimulation is an established therapy for the treatment of complex central and neuropathic pain syndromes refractory to medical treatment. Inevitably, ongoing evaluation will provide additional useful information regarding the surgical technique, initial indications, and long-term treatment effectiveness of MCS. In the last 10 years MCS has been shown to be an effective choice of treatment for patients who suffer without relief from this most difficult of pain syndromes.

\section{Disclosure}

This work is in publication (Brown, JA; Motor cortex stimulation, in Wilkins, RW, Rengachary, SS (eds): Neurosurgery, New York: McGraw-Hill, Vol 2, 2002.)

\section{References}

1. Andy OJ: Thalamic stimulation for chronic pain. Appl Neurophysiol 46:116-123, 1983

2. Bezard E, Boraud T, Nguyen JP, et al: Cortical stimulation and epileptic seizure: a study of the potential risk in primates. Neurosurgery 45:346-350, 1999

3. Ebel H, Rust D, Tronnier V, et al: Chronic precentral stimulation in trigeminal neuropathic pain. Acta Neurochir 138: 1300-1306, 1996

4. Garcia-Larrea L, Peyron R, Mertens P, et al: Electrical stimulation of motor cortex for pain control: a combined PET-scan and electrophysiological study. Pain 83:259-273, 1999

5. Garcia-Larrea L, Peyron R, Mertens P, et al: Positron emission tomography during motor cortex stimulation for pain control. Stereotact Funct Neurosurg 68:141-148, 1997

6. Gorecki J, Hirayama T, Dostrovsky JO, et al: Thalamic stimulation and recording in patients with deafferentation and central pain. Stereotact Funct Neurosurg 52:219-226, 1989

7. Hardy SG: Analgesia elicited by prefrontal stimulation. Brain Res 339:281-284, 1985

8. Hardy SG, Haigler HJ: Prefrontal influences upon the midbrain: a possible route for pain modulation. Brain Res 339:285-293, 1985

9. Hosobuchi Y: Chronic brain stimulation for the treatment of intractable pain. Res Clin Stud Headache 5:122-126, 1978

10. Hosobuchi Y: Subcortical electrical stimulation for control of intractable pain in humans. Report of 122 cases (1970-1984). J Neurosurg 64:543-553, 1986

11. Katayama Y, Fukaya C, Yamamoto T: Poststroke pain control by chronic motor cortex stimulation: neurological characteristics predicting a favorable response. J Neurosurg 89:585-591, 1998

12. Katayama Y, Tsubokawa T, Yamamoto T: Chronic motor cortex stimulation for central deafferentation pain: experience with bulbar pain secondary to Wallenberg syndrome. Stereotact Funct Neurosurg 62:295-299, 1994

13. Lende RA, Kirsch WM, Druckman R: Relief of facial pain after combined removal of precentral and postcentral cortex. J Neurosurg 34:537-543, 1971 


\section{Motor cortex stimulation}

14. Lenz FA, Kwan HC, Dostrovsky JO, et al: Characteristics of the bursting pattern of action potentials that occurs in the thalamus of patients with central pain. Brain Res 496:357-360, 1989

15. Melzack R: The McGill Pain Questionnaire: major properties and scoring methods. Pain 1:277-299, 1975

16. Melzack R, Terrence C, Fromm G, et al: Trigeminal neuralgia and atypical facial pain: use of the McGill Pain Questionnaire for discrimination and diagnosis. Pain 27:297-302, 1986

17. Meyerson BA, Lindblom U, Linderoth B, et al: Motor cortex stimulation as treatment of trigeminal neuropathic pain. Acta Neurochir Suppl 58:150-153, 1993

18. Namba S: [Role of the lemniscal system in pain modulation-a consideration based on clinical experience.] No Shinkei Geka 13:855-864, 1985 (Jpn)

19. Namba S, Shimizu Y, Wani T, et al: [An experimental model for deafferented pain in cats. Considerations based on neurochemical and electrophysiological findings]. Neurol Med Chir 25:715-722, 1985 (Jpn)

20. Nguyen JP, Keravel Y, Feve A, et al: Treatment of deafferentation pain by chronic stimulation of the motor cortex: report of a series of 20 cases. Acta Neurochir Suppl 68:54-60, 1997

21. Peyron R, Garcia-Larrea L, Deiber MP, et al: Electrical stimulation of precentral cortical area in the treatment of central pain: electrophysiological and PET study. Pain 62:275-286, 1995

22. Rainov NG, Fels C, Heidecke V, et al: Epidural electrical stimulation of the motor cortex in patients with facial neuralgia. Clin Neurol Neurosurg 99:205-209, 1997
23. Tsubokawa T, Katayama Y, Yamamoto T, et al: Chronic motor cortex stimulation in patients with thalamic pain. J Neurosurg 78:393-401, 1993

24. Tsubokawa T, Katayama Y, Yamamoto T, et al: Chronic motor cortex stimulation for the treatment of central pain. Acta Neurochir Suppl 52:137-139, 1991

25. Tsubokawa T, Katayama Y, Yamamoto T, et al: Deafferentation pain and stimulation of the thalamic sensory relay nucleus: clinical and experimental study. Appl Neurophysiol 48: $166-171,1985$

26. Tsubokawa T, Katayama Y, Yamamoto T, et al: Treatment of thalamic pain by chronic motor cortex stimulation. Pacing Clin Electrophysiol 14:131-134, 1991

27. White JC, Sweet WH: Pain: Its Mechanisms and Neurosurgical Control. Springfield, IL: Charles C Thomas, 1955

28. Yamamoto T, Katayama Y, Hirayama T: Pharmacological classification of central post-stroke pain: comparison with the results of chronic motor cortex stimulation therapy. Pain 72: 5-12, 1997

Manuscript received August 1, 2001.

Accepted in final form August 20, 2001.

Address reprint requests to: Jeffrey A. Brown, M.D., Department of Neurological Surgery, Wayne State University, School of Medicine, 4160 John R Suite 930, Detroit, Michigan 48201. email: jbrown@neurosurgery.wayne.edu. 